\title{
An Atmospheric Phase Screen Estimation Strategy Based on Multi-Chromatic Analysis for Differential Interferometric Synthetic Aperture Radar
}

\author{
Filippo Biondi, Member, IEEE, Carmine Clemente, Senior Member, IEEE, and Danilo Orlando, Senior Member,
} IEEE,

\begin{abstract}
In synthetic aperture radar (SAR) the separation of the height between the ground subsidence phase components and the atmospheric phase delay mixed in the global SAR interferometry (InSAR) phase information is an issue of primary concern in the remote sensing community. This paper describes a complete procedure to address the challenge to estimate the atmospheric phase screen and to separate the three-phase components by exploiting only one InSAR image couple. This solution has the capability to process persistent scatterers subsidence maps potentially using only two multi-temporal InSAR couples observed in any atmospheric condition. The solution is obtained by emulating the atmosphere compensation technique which is largely used by the global positioning system where two frequencies are used in order to estimate and compensate the positioning errors due to atmosphere parameters variations. A sub-chirping and sub-Doppler algorithm for athmosferic compensation is proposed which allows the successful separation of the height from the subsidence and of the atmosphere parameters from the interferometric phase observed on one InSAR couple. Results are given processing images of two InSAR couples observed by the COSMO-SkyMed satellite system.
\end{abstract}

Index Terms-Synthetic aperture radar, Atmospheric Phase Screen, Differential Interferometry, Multi-Chromatic analysis, MCA-APS-DInSAR.

\section{INTRODUCTION}

$\mathbf{S}$ YNTHETIC aperture radar (SAR) is a very precise space sensor used for earth subsidence estimation [1]. The most fruitful techniques for this operational task is the use of differential SAR interferometry (DInSAR), which is a multi-temporal extension of the interferometric SAR (InSAR). DInSAR measures Earth deformation with millimeter accuracy considering the flattened and unwrapped phase interferograms difference generated by two couples of InSAR images. If an Earth deformation occurs between this coherent multitemporal observations, it is possible to measure the subsidence generated by this event [2]- [3]. In [2]- [4]- [5]- [6] and [7] advanced DInSAR approaches have been developed to study the temporal evolution of the Earth displacement also

Filippo Biondi is with Italian Ministry of Defence. E-mail: biopippo@gmail.com.

Carmine Clemente is with the University of Strathclyde, Department of Electronic and Electrical Engineering, 204 George Street, G1 1XW, Glasgow, Scotland.

D. Orlando is with the Faculty of Engineering, Università degli Studi "Niccolò Cusano", 00166 Roma, Italy. E-mail: danilo.orlando@unicusano.it.

Carmine Clemente's work was supported by the Engineering and Physical Sciences Research Council (EPSRC) Grant number EP/K014307/1 and the MOD University Defence Research Collaboration in Signal Processing. using a reduced number of interferometric temporal SAR series [8] [9]. The problem of DInSAR is that a precise SAR interferometric phase compensation due to the atmospheric perturbation is highly required [10]- [11].

This paper is aimed at exploring a new procedure to provide an alternative solution to the one introduced in [2] to estimate a reliable atmospheric phase screen (APS) and to discriminate from the global InSAR observed phase, the component given by the topographic height, the phase contribution generated by the atmosphere and the component recorded by the Earth subsidence. The last task is a severe problem since the atmosphere is a medium that greatly disturbs the interferometric phase and this occurs in an uncontrollable manner [2]. This separation problem is solved by processing a long temporal series of interferometric SAR observations and the solution is given for highly coherent radar targets. The resulting procedure is based on inverting statistical models where it is necessary to deal with the correlation between the unwrapped phase residuals and the water vapor content of the atmosphere. Note that the latter is often the most important cause of artifacts in SAR interferograms [12]- [13]- [14]. The authors of [2] demonstrated the feasibility to estimate submeter DEM accuracy and millimetric terrain motion detection once the APS contributions have been estimated and removed. In this context, the present paper proposes a new approach to APS estimation. The technique invokes a direct measurement of the atmospheric phase delay by superimposing a carrier and Doppler frequency variation. The solution is found emulating the atmosphere compensation technique which is largely used by the global positioning system (GPS) where two frequencies are used in order to estimate and compensate the positioning errors due to atmosphere parameters variations [15]- [16]. A sub-chirping and sub-Doppler atmospheric compensation algorithm is derived which allows the successful separation of the height from the Earth movements and the atmosphere parameters from the interferometric phase observed on one InSAR couple. The effectiveness of the new proposed approach is validated using two InSAR couples acquired by the COSMO-SkyMed (CSK) satellite system.

The remainder of this paper is organized as follows. In the next section, a detailed description of the ideas behind the proposed procedure is provided, whereas in Sections III and IV the theoretical model for the SAR phase along with the effects on the interferometric SAR measurements due to the electromagnetic SAR propagation through the the ionosphere 
and the troposphere are presented. Section V is devoted to the definition of the Multi-Chromatic Analysys (MCA) sub-apertures architecture for DInSAR compensation and the experimental results. Finally, some concluding remarks and future research tracks are given in Section VI.

\section{Methodology}

The retrieval of topographic Earth deformation has been one of the most active research fields since the beginning of the InSAR theory. The APS estimation and removal permits to precisely measure Earth deformation [2]. In a SAR image the pixel phase information changes its value mainly due to the line of sight (LOS) variations between satellite and target during the flight evolution. These variations can be also induced by time-varying target positions or by some changes of the atmospheric parameters spread over the SAR image, observed on the same temporal event. This phenomenon is called the spatial distribution of the atmospheric radar phase contribution. If an interferometric couple of SAR data is acquired, the global spatial APS of the InSAR product is affected by the spatial atmospheric contribution of each SAR image which differs one from the other by a temporal variation of this repeat-pass InSAR configuration. Long temporal series which are designed for persistent monitoring of sensible targets having millimeter space variation need the estimation of the APS for each temporal interferometric coherence. Fig. 1 shows the characteristics of the InSAR phase deviation versus pressure and relative humidity. The characteristics are plotted for different bands (X,C,L) and demonstrate how critical is the need for an accurate APS estimation. This processing stage is fundamental and so far has been addressed only throuhg the processing of a long temporal series [2].

This research is aimed at detecting the APS of each smalltemporal interferogram coherence independently, even though the series is composed by only one InSAR couple. To this end, the atmosphere compensation technique which is largely used by the GPS is exploited to remove the APS. Specifically, Two different frequencies are required for compensation and are generated by sub-chirping and sub-Doppler refocusing the InSAR couples. MCA uses interferometric pairs of SAR images processed at range subbands and explores the phase trend of each pixel as a function of the different central carrier frequencies to infer absolute optical path difference [17]. Space satellites positioning systems like GPS and Galileo offer facilities for accurate three-dimensional position corrections for both navigational and geodetic purposes. The signals from these satellites, when propagated through the ionosphere, are subject to phase delay, refraction, and dispersion [18]. It is important to note that a dual-frequency compensation can remove first-order ionospheric and tropospheric effects, while in a single-frequency GPS, range-measurement errors are mainly due to uncompensated ionospheric GPS-signal time delay [19]. In particular, a dual-frequency GPS computes the ionospheric error compensation to range error by measuring the difference in time delays between the two operating frequencies. For such applications, some augmentation processes are required. However, the constant motion of a remote sensing
SAR low-Earth orbit (LEO) satellite requires to consider Doppler frequency shifts useful to synthesize the image in the slow-time direction. When the same SAR raw data is focused using different electromagnetic chirp bands, this shift in frequency arises. Typically a LEO satellite motion with respect to an Earth-fixed observer can result in a maximum range of approximately $25 \mathrm{kHz}$ in Doppler band with respect to the electromagnetic carrier set at $10 \mathrm{GHz}$. Integration of the Doppler frequency offset results in an extremely accurate measurement of the increment in signal carrier phase between time epochs. Interferometric techniques between spatial and frequency baselines take advantages of these precise phase measurements in building-up reliable spatial-APS (SAPS) and temporal-APS (TAPS) useful for extremely precise Earth surface movement estimation.

\section{Electromagnetic SAR Propagation Through THE ATMOSPHERE}

The Earth atmosphere is characterized to have higher index of refraction than the free space. This phenomenon causes that the electromagnetic propagation velocity is lowered with respect to the light speed measured in the vacuum. The electric component of the complex SAR electromagnetic plane wave field is given by the following expression:

$$
E(x, t)=\exp \{j(k x-\omega t)\},
$$

where $j$ is the imaginary unit, $k=2 \pi n(x) / \lambda$ is the wavenumber with being $n(x)$ and $\lambda$ the refraction index and the operating wavelength, respectively, and $\omega=2 \pi f_{c}$ with $f_{c}$ the carrier frequency. The electromagnetic phase differential with respect to the path length $d x$ is $d \varphi=(2 \pi n(x) / \lambda) d x$. It follows that in free space, namely for $n(x)=1$, the entire phase variation along the satellite-target LOS is [21]

$$
\Delta \varphi=\int_{\Omega_{x}} \frac{2 \pi n(x)}{\lambda} d x=\frac{2 \pi}{\lambda} \Delta_{x},
$$

where $\Omega_{x}$ is the satellite-target propagation path and $\Delta_{x}$ is its length. Thus, in free space the electromagnetic phase shift $\Delta \phi$ depends only on $\lambda$ and $\Delta_{x}$. However, when the propagation space is filled by the atmosphere matter, $n(x)$ is not linear and the diffraction perturbation generates different orders of additional phase shifts. During the SAR acquisition at each electromagnetic received burst, a fractional phase measurement is made by the receiver through the carrierphase tracking-loop located along the decoding chain. The atmospheric phase delays can be decomposed into two main components namely the ionosphere and the troposphere contributions. The former is proportional to the total electrons content (TEC) along the transmit and receive electromagnetic path. This delay is denoted by $\epsilon^{I o n o}$ and can be estimated as

$$
\epsilon^{\text {Iono }}=\frac{2}{\sin \phi} \cdot \frac{40.3}{f_{c}^{2}} \cdot \text { TEC. }
$$

where $\phi$ is the incidence angle with respect to the vertical at the surface point. The term $\sin \phi$ is representative of the additional path length in the ionosphere when the electromagnetic LOS is vertical. The thickness of the ionosphere layer is typically variable from 300 to $400 \mathrm{~km}$. 
Equation 3 can be further modified to account for the SAR geometry and using the SAR reference system whose origin is located on the satellite. Specifically, the slant range additional path becomes

$$
\begin{aligned}
\left|\epsilon_{\text {slant }}^{\text {Iono }}\right| & =F_{P P} \cdot \frac{40.3 \mathrm{TEC}}{f^{2}}, \\
F_{p p} & =\left[1-\left(\frac{R_{e} \cos \theta}{R_{e}+h_{I}}\right)^{2}\right]^{-\frac{1}{2}},
\end{aligned}
$$

where $R_{e}$ is the radius of the Earth, $h_{I}$ is the distance between the Earth surface and the ionospheric bottom layer (which is typically $350 \mathrm{~km}$ ), and $\theta$ is the elevation angle of the SAR acquisition in the slant vector space reference. Parameter TEC takes on values in between $10^{16}$ and $10^{18}$ electrons $/ \mathrm{m}^{2}$. For the particular composition of the Earth tropospheric atmosphere, the electromagnetic phase shift can be expressed as

$$
\begin{aligned}
\Delta \varphi & =\frac{2 \pi}{\lambda} \Delta x+\frac{2 \pi 10^{-6} N(\Delta x)}{\lambda} \Delta x \\
& =\frac{2 \pi}{\lambda}[\Delta x+\delta(\Delta x)],
\end{aligned}
$$

where $N(\Delta x)$ is an additional refractive index generated by the atmosphere and $\delta(\Delta x)=10^{-6} N(\Delta x) \Delta x$ represents an incremental path length yielding an additional phase shift. According to [22], the latter contribution can be decomposed accounting for the water vapor and hydrostatic components as

$$
\delta(\Delta x)=\delta(\Delta x)_{W e t}+\delta(\Delta x)_{D r y} .
$$

Thus, given the slant nature of SAR geometry, it is possible to express the total additional two-way phase delay for sidelooking imaging radars as:

$$
\Delta \Delta \varphi=\frac{4 \pi}{\lambda} \cdot \frac{\delta(\Delta x)}{\cos \phi}
$$

where $\phi$ is the incidence angle. Fig. 1 depicts the electromagnetic phase delay versus the atmospheric pressure and relative humidity. The functions represent the integrated excess phase delay due to pressure and humidity variations computed according to (8) at several wavelengths (generated by chirp sub-apertures) and incidence angles (generated by Doppler sub-apertures).

\section{INTERFEROMETRIC SAR PHASE MEASUREMENTS UNDER ATMOSPHERIC EFFECTS}

Repeat-pass InSAR is designed for Earth topography estimation. The system geometry consists of two radars $S_{1}$ and $S_{2}$ which are spatially separated by a physical baseline. According to the repeat-pass characteristic of the InSAR observation, it is possible to use only one radar where acquisitions are performed at a different time and preserving the same geometry. The interval between two interferometric opportunities is equal to the orbital cycle which can take several days. A schematic representation of the InSAR acquisition is depicted in Fig. 2 (a). In the course of an InSAR acquisition, it may occur a variation of the Earth topography as depicted in Fig. 2 (b), where $\delta z$ represents the variation in height of the topography between two InSAR acquisitions. In the same figure, $B_{\|}$and $B_{\perp}$ are the parallel and the orthogonal baseline components, respectively, whereas $\theta$ and $\alpha$ are the look and baseline orientation angles, respectively. Now, focus on Fig. 2 (a), where no Earth topography variation has been generated and note that if two path lengths are denoted by $\rho$ and $\rho+\delta \rho$, the measured interferometric phase difference $d_{\varphi}$ is

$$
d_{\varphi}=\frac{4 \pi}{\lambda} \delta \rho
$$

When the radar-targets distance $\rho$ is much longer than the interferometric baseline $B$ it is possible to consider the electromagnetic rays nearly parallel. According to this approximation, the difference in length $\delta \rho$ is equal to:

$$
\delta \rho=B \sin (\theta-\alpha) \text { or } \delta \rho=B_{\|} .
$$

In (10) the parameter $B_{\|}=B \sin (\theta-\alpha)$ is the parallel baseline component to the look direction. Recall that it is possible to infer the topography exploiting precise measurements of the look-angle $\theta$ at each azimuth-range resolution cell [21], namely

$$
z=h-\rho \cos \theta
$$

where the parameter $h$ is the height of the SAR platform.

In order to observe some Earth deformation activity it is necessary to perform a second interferogram acquired over the same region but after the Earth perturbation event (Fig. 2 (b)). The considered perturbation will generate a displacement which is assumed to be small withl respect to the radar resolution cell. The new post-event interferogram which is supposed to have the same geometry will instead measure a different phase which is equal to:

$$
d_{\varphi_{\text {Post-event }}}=\frac{4 \pi}{\lambda} \delta \rho_{\text {Post-event }},
$$

where $\delta \rho_{\text {Post-event }}=B_{\|}+\delta z$ and $\delta z$ yields a sub-wavelength sensitivity motion. In order to measure the deformation, it is necessary to remove the topographic component by subtracting a precise DEM of the same observed area. The real world is embedded in a space filled with the atmosphere having a thickness of approximately $450 \mathrm{~km}$ so all interferograms are formed using signals that have propagated through the atmosphere. According to this problem, it is necessary to examine in detail the effects of propagation delay variations on DInSAR deformation maps. To solve this problem, a DInSAR solution using a three-pass interferometric SAR configuration for surface Earth deformation measurement is herein devised. The proposed method will perform a precise APS estimation by observing a single interferometric couple. It is important to recall that the estimated height error given by [21]

$$
\sigma_{z}=\frac{\lambda \rho}{4 \pi B} \frac{\sin \theta}{\cos (\theta-\alpha)} \sigma_{\theta},
$$

where $\sigma_{\theta}$ is the look angle error in the interferogram, is a function of the geometry, operating frequency, and atmospheric variability. Thus, the main idea of this single-pair interferometric APS estimation procedure is that generating a frequency perturbation in the SAR observation, it is possible 
to discriminate between the interferometric phase delay due to the atmosphere and the interferometric phase variation due to Earth deformation. In particular, assuming that ground deformation is invariant to frequency variations, the latter can be separated from the atmosphere contributions to the phase delay. The frequency perturbation is generated using the MCA [17]. The technique uses interferometric SAR images which are split into range sub-bands in order to study the phase trend of each pixel as a function of the different central carrier frequencies. Moreover, MCA is suitably modified to create sub-bands also in the Doppler domain and guarantee an enhanced APS estimation quality. Remarkably, the proposed procedure can operate exploiting small-temporal interferogram coherence independently, even if the image series consists of only one InSAR pair.

\section{Processing Architecture And Experimental RESULTS}

In this section, the proposed processing architecture is described in the detail and the resulting performance are investigated resorting to two study cases. The first illustrative examples rely on a SAR image representing the environment of the Mosul dam in the Iraq state. The Region of Interest (RoI) of the second study case is located in the state of Libya.

\section{A. Processing Scheme}

Fig. 3 contains a pictorial description of the architecture for APS estimation in terms of a block-scheme, which is formed by 51 blocks. The scheme is fed by three raw SAR images, one master and two slaves represented by blocks with labels from 1 to 4 . Raw data undergo a low-pass and highpass filtering (blocks numbered from 5 to 10) to create the sub-apertures. Note that the figure refers to the processing along the range dimension. As for the azimuth dimension, the required steps are analogous. For this reason, the description below considers the range dimension only. Before including a precise orbit phase error compensation, all the sub-apertures are processed by the range-azimuth focusing computational blocks with numbers $17,18,20,21,23,24,26$, and 27. Moreover, the full-band images are also focused using blocks with numbers $19,22,25$, and 28 . The outputs of these blocks are the full-resolution SAR images which are used to create the uncompensated Digital Elevation Models (DEM)s. Computational blocks 29, 30, 32, and 33 perform the flatEarth interferometric phase estimation and removal for the chirp/Doppler sub-aperture products, whereas computational blocks 31 and 34 repeat the same operations for the fullband images. The phase unwrapping lies in the computational stages $35,36,38,39$ (sub-aperture products), and 38 (full-band products). An uncompensated coarse Earth deformation is now available by computing the (DEM) difference between the outputs of blocks number 46 and 43 . Blocks number 41, 42, 44, 45 (sub-aperture products) and 43, 46 (full-band products) are devoted to the geocoding of DEMs.
Then, the following atmosphere maps are evaluated as (blocks 47-50)

$$
\begin{aligned}
& \mathrm{APS}_{1}^{R}=\angle\left\{\exp \left[j\left(\mathrm{DEM}_{2}^{R}-\mathrm{DEM}_{1}^{R}\right)\right]\right\} \\
& \mathrm{APS}_{2}^{R}=\angle\left\{\exp \left[j\left(\mathrm{DEM}_{4}^{R}-\mathrm{DEM}_{3}^{R}\right)\right]\right\} \\
& \mathrm{APS}_{3}^{R}=\angle\left\{\exp \left[j\left(\mathrm{DEM}_{3}^{R}-\mathrm{DEM}_{1}^{R}\right)\right]\right\} \\
& \mathrm{APS}_{4}^{R}=\angle\left\{\exp \left[j\left(\mathrm{DEM}_{4}^{R}-\mathrm{DEM}_{2}^{R}\right)\right]\right\},
\end{aligned}
$$

where $\angle\{\cdot\}$ returns the phase of the complex argument. Additional atmosphere maps are formed by repeating the same processing along the Doppler dimension, namely

$$
\begin{aligned}
& \operatorname{APS}_{1}^{D}=\angle\left\{\exp \left[j\left(\mathrm{DEM}_{2}^{D}-\mathrm{DEM}_{1}^{D}\right)\right]\right\} \\
& \mathrm{APS}_{2}^{D}=\angle\left\{\exp \left[j\left(\mathrm{DEM}_{4}^{D}-\mathrm{DEM}_{3}^{D}\right)\right]\right\} \\
& \operatorname{APS}_{3}^{D}=\angle\left\{\exp \left[j\left(\mathrm{DEM}_{3}^{D}-\mathrm{DEM}_{1}^{D}\right)\right]\right\} \\
& \mathrm{APS}_{4}^{D}=\angle\left\{\exp \left[j\left(\mathrm{DEM}_{4}^{D}-\mathrm{DEM}_{2}^{D}\right)\right]\right\}
\end{aligned}
$$

where $\operatorname{APS}_{i}^{D}$ and $\operatorname{DEM}_{i}^{D}, i=1, \ldots, 4$, are the analogous counterparts along the Doppler dimension of $\operatorname{APS}_{i}^{R}$ and $\mathrm{DEM}_{i}^{R}, i=1, \ldots, 4$. The compensated wrapped interferograms are given by

$$
\begin{aligned}
& \text { Int }_{\text {Comp pre }}=\angle \exp \left\{j\left[\angle\left(S_{1} \cdot S_{2}^{*}\right)-\mathrm{APS}_{1}^{R}-\mathrm{APS}_{1}^{D}\right]\right\},
\end{aligned}
$$

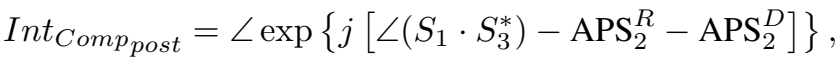

$$
\begin{aligned}
& \delta_{z \text { wrapped }}=\angle\left\{j\left(\text { Int }_{\text {Comp }} \text { pre }- \text { Int }_{\text {Comp post }}\right)\right. \\
& \left.-\mathrm{APS}_{3}^{R}-\mathrm{APS}_{3}^{D}-\mathrm{APS}_{4}^{R}-\mathrm{APS}_{4}^{D}\right\} \text {. }
\end{aligned}
$$

The compensated DEM difference which contains only the Earth deformation is equal to:

$$
\begin{gathered}
\mathrm{DEM}_{C o m p_{p r e}}=\mathrm{DEM}_{F u l l 1}-\mathrm{DEM}_{A P S_{1}^{R}}-\mathrm{DEM}_{A P S_{1}^{D}}, \\
\mathrm{DEM}_{C o m p_{p o s t}}=\mathrm{DEM}_{F u l l 2}-\mathrm{DEM}_{A P S_{2}^{R}}-\mathrm{DEM}_{A P S_{2}^{D}}, \\
\delta_{z}=\mathrm{DEM}_{C o m p_{p r e}}-\mathrm{DEM}_{C o m p_{p o s t}}-\mathrm{DEM}_{A P S_{3}^{R}} \\
-\mathrm{DEM}_{A P S_{3}^{D}}-\mathrm{DEM}_{A P S_{4}^{R}}-\mathrm{DEM}_{A P S_{4}^{D}} .
\end{gathered}
$$

In the last equations, $\mathrm{DEM}_{C o m p}{ }_{\text {pre }}$ is the compensated preevent DEM result, $\mathrm{DEM}_{\text {Comp }}$ post is the compensated postevent DEM, and $\mathrm{DEM}_{A P S_{i}^{X}}, X \in\{R, D\}$ and $i=1, \ldots, 4$, are the heights corresponding to phase maps $\operatorname{APS}_{i}^{X}$. Finally, equation (14) represents the real Earth deformation result cleaned from all the atmospheric artifacts which generate a dramatic amount of false alarms on the subsidence map.

\section{B. Numerical Examples and Discussion}

This section shows the effectiveness of the previously described processing scheme over two sets of data. The chirp band of the strip map image of CSK is $96 \mathrm{MHz}$ while the Doppler band is of $26 \mathrm{kHz}$. The spatial resolution is $3 \mathrm{~m}$ in the range and in the azimuth directions. The temporal scheduling of the interferometric SAR acquisitions for both the study cases is depicted in Fig. 4 (a). The frequency allocation plane is described in Fig. 4 (b). Two sub-apertures in the fast time domain and other two in the Doppler domain have been designed to estimate the APS. 


\begin{tabular}{|c|c|c|c|}
\hline Date & Type & Pol. & IA $\left(^{\circ}\right)$ \\
\hline $07-10-2013$ & Stripmap & $\mathrm{HH}$ & 35.317742 RA \\
\hline $23-10-2013$ & Stripmap & $\mathrm{HH}$ & 35.317552 RA \\
\hline $19-06-2013$ & Stripmap & $\mathrm{HH}$ & $35.317452 \mathrm{RA}$ \\
\hline $20-03-2016$ & Spotlight & $\mathrm{HH}$ & $40.517450 \mathrm{RA}$ \\
\hline 05-04-2016 & Spotlight & $\mathrm{HH}$ & $40.913052 \mathrm{RA}$ \\
\hline 21-04-2016 & Spotlight & $\mathrm{HH}$ & 40.217454 RA \\
\hline
\end{tabular}

TABle 1. SAR Data AdDitional Details

According to Fig. 4 (b), the first sub-aperture has been focused setting a chirp sub-band starting from $0 \mathrm{~Hz}$ to 53 $\mathrm{MHz}$ with a central frequency of $26.5 \mathrm{MHz}$ and using all the available Doppler band. The second sub-aperture SAR product has been focused setting processing the chirp frequencies starting from $43 \mathrm{MHz}$ to $96 \mathrm{MHz}$ with central frequency 69.5 $\mathrm{MHz}$ and using all the available Doppler band. The third subaperture was focused setting processing only all the Doppler spectrum starting from $0 \mathrm{~Hz}$ to $14 \mathrm{kHz}$ with a central frequency set to $6.5 \mathrm{kHz}$ and keeping all the available chirp band. The last sub-aperture was focused the rest of the Doppler sub-aperture available from $12 \mathrm{~Hz}$ to $26 \mathrm{kHz}$ on a central frequency set to $20.5 \mathrm{kHz}$ and processing all the available chirp band. Further details about the SAR platform are reported in I, where the first and the second columns of the table contain the acquisition date and type, respectively; the third column consider the polarization (note that only $\mathrm{HH}$ images have been processed) whereas in the fourth column the incidence angles are listed; finally, the last column of the table indicates the orbit type (note that only right ascending (RA) orbits were used). The master image of the first considered data set was observed on October the 7th 2013 and two slaves were acquired on October the 23rd 2013 and June the 19th 2015. As for the second study case, the master imagewas acquired on March the 20th 2013 while two slaves were observed on April the 5th 2016 and April the 21st 2016. The time existing between the first and the second slaves is sufficient to observe some deformations occurred on the dam (first study case) and some subsidences of the earth (second study case).

1) Study Case 1: With reference the to Fig. 2, the interferometric configuration used for acquisition before the Earthdeformation event, denoted by (07/10/2013-23/10/2013)-data, is described by subplot (a) while subplot (b) depicts the configuration for data collection after Earth-deformation event, referred to as (07/10/2013-19/06/2015)-data. This geometry performs three-pass DInSAR where the APS compensation is estimated using the information given by the frequency diversity. Fig. 5 is the single-look complex SAR image of the master acquisition; Fig. 6 is the flattened interferogram estimated by processing (07/10/2013-23/10/2013)-data representing the first InSAR product of the DInSAR system and where the unwrapped DEM is represented in Fig. 7. Fig. 8 is the flattened interferogram estimated processing the (07/October/2013-19/June/2015)-data repesenting the second InSAR product of the DInSAR system and where the unwrapped DEM is represented in Fig. 9. Fig. 10 are two elevation profiles observed by the two DEMs. The coarse Earth deformation estimation, which is computed by subtracting the full-band DEM corresponding to the first and the second InSAR acquisitions, is plotted in Fig. 11; the orthorectified version of the latter is provided in Fig. 12. This result contains a significant amount of artifacts due to the atmosphere density variations. These atmospheric turbulences due to the troposphere generates a large number of false alarms visible in red on the uncompensated Earth deformation map.

The main inputs useful to estimate the APS are the following DEMs:

- $\mathrm{DEM}_{\text {full }_{1}}$ estimated using (07/10/2013-23/10/2013)data, which are focused exploiting all the available chirpDoppler band;

- $\mathrm{DEM}_{\text {full }_{2}}$ estimated using (07/10/2013-19/06/2015)data, which are focused exploiting all the available chirpDoppler band;

- $\mathrm{DEM}_{1}^{R}$ estimated using (07/10/2013-23/10/2013)-data, which are focused using the first half chirp band (from 0 $\mathrm{MHz}$ to $53 \mathrm{MHz}$ );

- $\mathrm{DEM}_{2}^{R}$ estimated using (07/10/2013-23/10/2013)-data, which are focused using the second half chirp band (from $43 \mathrm{MHz}$ to $96 \mathrm{MHz}$ );

- $\mathrm{DEM}_{3}^{R}$ estimated using (07/10/2013-19/06/2013)-data, which are focused using the first half chirp band (from 0 $\mathrm{MHz}$ to $53 \mathrm{MHz}$ );

- $\mathrm{DEM}_{4}^{R}$ estimated using (07/10/2013-19/06/2013)-data, which are focused using the second half chirp band (from $43 \mathrm{MHz}$ to $96 \mathrm{MHz}$ );

- $\mathrm{DEM}_{1}^{D}$ estimated using (07/10/2013-23/10/2013)-data, which are focused using the first half Doppler band (from $0 \mathrm{MHz}$ to $14 \mathrm{kHz}$ );

- $\mathrm{DEM}_{2}^{D}$ estimated using (07/10/2013-23/10/2013)-data, which are focused using the second half Doppler band (from $12 \mathrm{MHz}$ to $26 \mathrm{kHz}$ );

- $\mathrm{DEM}_{3}^{D}$ estimated using (07/10/2013-19/06/2013)-data, which are focused using the first half Doppler band (from $0 \mathrm{MHz}$ to $14 \mathrm{kHz}$;

- $\mathrm{DEM}_{4}^{D}$ estimated using (07/10/2013-19/06/2013)-data, which are focused using the second half Doppler band (from $12 \mathrm{MHz}$ to $26 \mathrm{kHz}$ );

- $\mathrm{DEM}_{A P S_{1}^{R}}$ estimated unwrapping the $A P S_{1}^{R}$ phase map.

- $\operatorname{DEM}_{A P S_{2}^{R}}$ estimated unwrapping the $A P S_{2}^{R}$ phase map.

- DEM $_{A P S_{3}^{R}}$ estimated unwrapping the $A P S_{3}^{R}$ phase map.

- $\mathrm{DEM}_{A P S_{4}^{R}}$ estimated unwrapping the $A P S_{4}^{R}$ phase map.

- $\mathrm{DEM}_{A P S_{1}^{D}}$ estimated unwrapping the $A P S_{1}^{D}$ phase map.

- $\mathrm{DEM}_{A P S_{2}^{D}}$ estimated unwrapping the $A P S_{2}^{D}$ phase map.

- $\mathrm{DEM}_{A P S_{3}^{D}}$ estimated unwrapping the $A P S_{3}^{D}$ phase map.

- $\mathrm{DEM}_{A P S_{4}^{D}}$ estimated unwrapping the $A P S_{4}^{D}$ phase map.

The final results are visible in Fig. 12 where the uncompensated geocoded Earth deformation map is shown and in Fig. 21 containing the same representation but completely compensated. As an example, Fig. 13, 14, 15 and 16 are the representation of $A P S_{1}^{R}, A P S_{2}^{R}, A P S_{1}^{D}$ and $A P S_{2}^{D}$ respectively. Further details of the Earth subsidence highlighted by the two boxes drawn in Fig. 11 are provided in Figs. 17-20. Specifically, the uncompensated environment contained inside 
the blue box number one is magnified in Fig. 17 whereas the compensated result is represented by Fig. 18 which gives only the true movements occurring on the Earth surface. The environment contained inside the box number two of Fig. 11 is a small piece of Earth bordered by the water. The uncompensated results, provided in Fig. 19, contain very high levels of artifacts, on the contrary in Fig. 20, where the APS compensation is applied, no artifacts are visible. Finally, in Fig. 22, the uncompensated and compensated height profiles of the region contained in the red box of Fig. 20 are shown. It turns out that uncompensated curve exhibits a chaotic trend which does not provide any information. On the other hand, the compensated profile is almost flat except for in the last pixels where an absidence raises with high probability.

2) Study Case 2: The second study case considers the processing of three spotlight images. This configuration of SAR acquisitions permits the observation of the Earth with a spatial resolution of approximately one meter along the range and azimuth directions. The chirp and Doppler bands are perfectly partitioned into two equally distributed subapertures without any kind of overlap. Fig. 23 is the geocoded uncompensated DInSAR result where it is possible to view the effects of the atmosphere which prevent the operator from any kind of terrain deformation analysis. Fig. 24 contains the geocoded and compensated results using the proposed MCA-APS technique and, interestingly, the area contained in the red box is magnified in Fig. 25 where some light deformations due to human activities (red circles indicated by the blue arrows number 1,2 and 3) are clearly visible in a extremely stable background (recall that the used pair of InSAR images are temporally separated by less than 1 month). In the last four figures, the compensation without band overlap is compared with that in the presence of a overlap of about $20 \%$. Specifically, Fig. 26 contains the uncompensated map, Fig. 27 refers to the APS compensation for the area in the red box of Fig. 26 in the presence of overlap, and in Fig. 28 the compensation (for the same region) is applied without band overlap. Finally, Fig. 29 shows the height profiles for the region highlighted by the horizontal red line of Fig. 26. As expected the figures point out that the compensation without overlap is more effective than the compensation in the presence of band overlap. As a matter of fact, the Root Mean Square (RMS) value of the difference between the uncompensated map and the map compensated with overlap returns 1.3288 , while the RMS value of the difference between the uncompensated map and the map compensated without overlap returns 3.4144 meaning that, in the latter case, the level of compensation is higher with respect to the case with overlap.

Summarizing, this second datasets confirms the effectiveness of the proposed MCA-APS compensation technique.

\section{CONCLUSIONS}

In this paper, an innovative procedure for compensation of APS has been proposed. Remarkably, it can operate exploiting only two multi-temporal InSAR couples acquired in any atmospheric condition. The main idea relies on the atmosphere compensation technique which is largely used by the GPS where two frequencies are used in order to estimate and compensate the positioning errors due to atmosphere parameters variations. To this end, a sub-chirping and Doppler atmospheric compensation algorithm has been conceived to to successfully separate the height from the subsidence and the atmosphere parameters from the interferometric phase observed on one InSAR couple. The effectiveness of the entire procedure has been validated by applying the proposed approach on two sets of data. Numerical examples have highlighted that the height profiles generated by the newly proposed procedure using only a pair of InSAR images are more reliable than those produced without compensating the deleterious APS effects.

Finally, future research tracks may include the design of an iterative compensation procedure that exploits more than a pair of InSAR images aimed at improving the compensation performance of the herein proposed procedure.

\section{REFERENCES}

[1] Gabriel, Andrew K.; Goldenstein, Richard M.; Zebker, Howard A. Mapping small elevation changes over large areas: differential radar interferometry. Journal of Geophysical Research: Solid Earth, 1989, 94.B7: 9183-9191.

[2] Ferretti, Alessandro; Prati, Claudio; Rocca, Fabio. Permanent scatterers in SAR interferometry. IEEE Transactions on geoscience and remote sensing, 2001, 39.1: 8-20.

[3] Massonet, Didier, et al. The displacement field of the Landers earthquake mapped by radar interferometry. Nature, 1993, 364.6433: 138.

[4] Ferretti, Alessandro; Prati, Claudio; Rocac, Fabio. Nonlinear subsidence rate estimation using permanent scatterers in differential SAR interferometry. IEEE Transactions on Geoscience and Remote Sensing, 2000, 38.5: 2202-2212.

[5] Bernardino, Paolo, et al. A new algorithm for surface deformation monitoring based on small baseline differential SAR interferograms. IEEE Transactions on Geoscience and Remote Sensing, 2002, 40.11: 2375-2383.

[6] Bernardino, Paolo, et al. A new algorithm for surface deformation monitoring based on small baseline differential SAR interferograms. IEEE Transactions on Geoscience and Remote Sensing, 2002, 40.11: 2375-2383.

[7] Casu, Francesco, et al. SBAS-DInSAR Analysis of Very Extended Areas: First Results on a 60 000-km2 Test Site. IEEE Geosci. Remote Sensing Lett., 2008, 5.3: 438-442.

[8] Casu, Francesco, et al. "Deformation time-series generation in areas characterized by large displacement dynamics: The SAR amplitude pixel-offset SBAS technique." IEEE Transactions on Geoscience and Remote Sensing 49.7 (2011): 2752-2763.

[9] Casu, Francesco, and Andrea Manconi. "Four-dimensional surface evolution of active rifting from spaceborne SAR data." Geosphere 12.3 (2016): 697-705.

[10] Monti-Guarnieri, A., et al. SAR interferometry experiments with ERS1. In: Geoscience and Remote Sensing Symposium, 1993. IGARSS'93. Better Understanding of Earth Environment., International. IEEE, 1993. p. 991-993.

[11] Wang, Zhiying, Daniele Perissin, and Hui Lin. "Subway tunnels identification through Cosmo-SkyMed PSInSAR analysis in Shanghai." Geoscience and Remote Sensing Symposium (IGARSS), 2011 IEEE International. IEEE, 2011.

[12] Rodon, Josep Ruiz, et al. Geosynchronous SAR Focusing With Atmospheric Phase Screen Retrieval and Compensation. IEEE Trans. Geoscience and Remote Sensing, 2013, 51.8: 4397-4404.

[13] Perissin, Daniele, et al. Mitigation of Atmospheric Water-vapour Effects on Spaceborne Interferometric SAR Imaging through the MM5 Numerical Model. PIERS Online, 2010, 6.3: 262-266.

[14] Pierdicca, Nazzareno, et al. Atmospheric water vapor effects on spaceborne interferometric SAR imaging: Comparison with ground-based measurements and meteorological model simulations at different scales. In: Geoscience and Remote Sensing Symposium, 2009 IEEE International, IGARSS 2009. IEEE, 2009. p. V-320-V-323. 
[15] Ajayi, G. O.; Hedberg, A.; Hamberg, Gunno. Accurate determination of ionospheric effects on satelliteâbased positioning systems in terms of residual range error. Radio Science, 1980, 15.5: 1009-1016.

[16] Feess, W. A.; Stephens, S. G. Evaluation of GPS ionospheric time-delay model. IEEE Transactions on Aerospace and Electronic Systems, 1987, 3: 332-338.

[17] Bovenga, Fabio, et al. Multichromatic analysis of InSAR data. IEEE Transactions on Geoscience and Remote Sensing, 2013, 51.9: 47904799.

[18] AJAYI, G. O.; Hedberg, A.; Hamberg, Gunno. Accurate determination of ionospheric effects on satelliteâbased positioning systems in terms of residual range error. Radio Science, 1980, 15.5: 1009-1016.

[19] Feess, W. A.; Stephens, S. G. Evaluation of GPS ionospheric time-delay model. IEEE Transactions on Aerospace and Electronic Systems, 1987, 3: 332-338.

[20] Deng, Zhiguo, et al. Validation of tropospheric slant path delays derived from single and dual frequency GPS receivers. Radio Science, 2011, 46.06: $1-11$

[21] Zebker, Howard A.; Rosen, Paul A.; Hensley, Scott. Atmospheric effects in interferometric synthetic aperture radar surface deformation and topographic maps. Journal of Geophysical Research: Solid Earth, 1997, 102.B4: 7547-7563.

[22] Goldhirsh, Julius; Rowland, John R. A tutorial assessment of atmospheric height uncertainties for high-precision satellite altimeter missions to monitor ocean currents. IEEE Transactions on Geoscience and Remote Sensing, 1982, 4: 418-434.

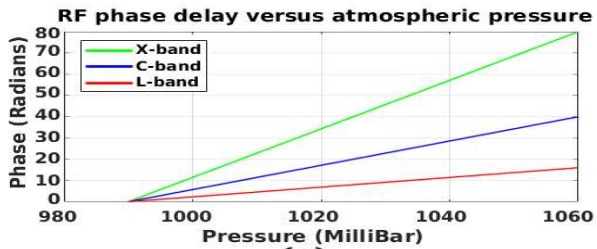

(a)

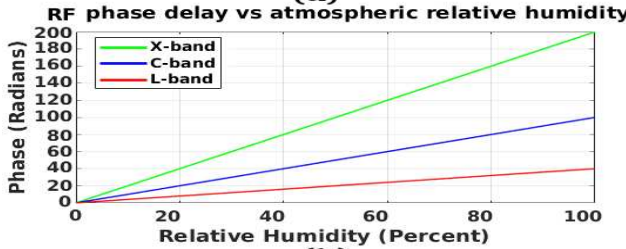

(b)

Fig. 1. Pressure versus phase (a) and Relative humidity versus phase (b).

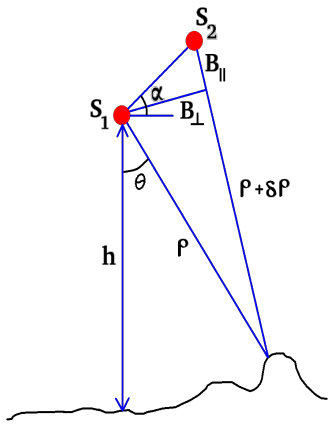

InSAR acquisition at time T1

(a)

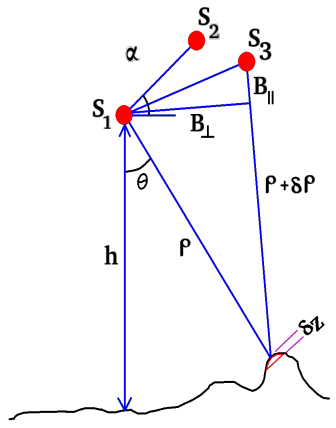

InSAR acquisition at time $\mathrm{T} 2$ (b)

Fig. 2. DInSAR acquisition temporal geometry. (a): InSAR geometry for time T1. (b): InSAR geometry for time T2.
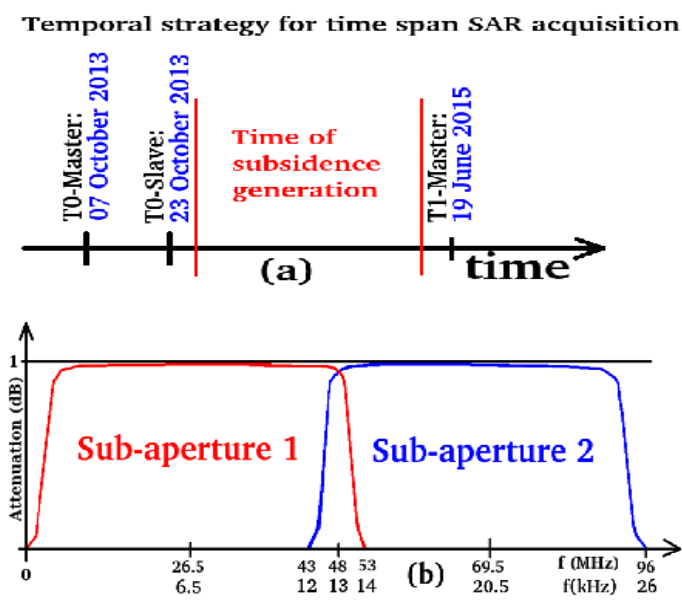

Fig. 4. (a): SAR acquisition time history. (b): Chirp-Doppler frequencies allocation planes.

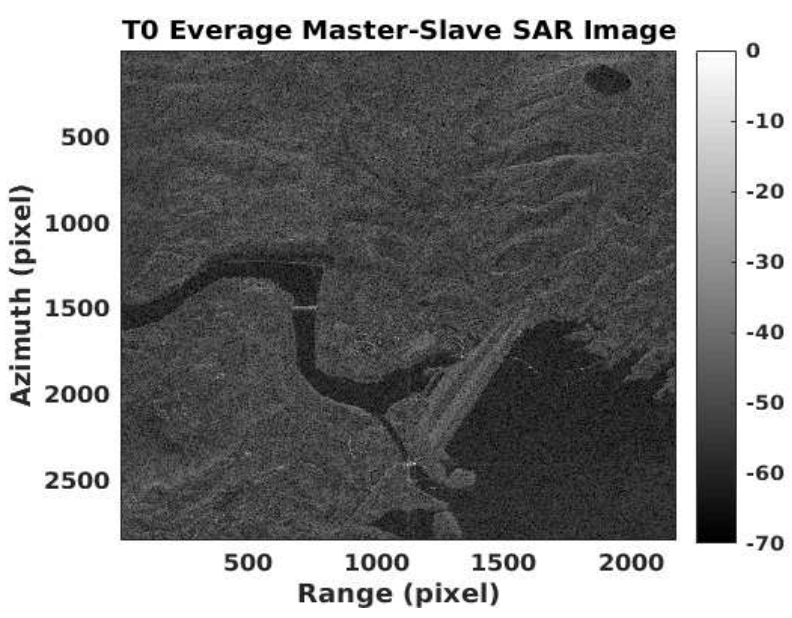

Fig. 5. SLC RoI (logarithmic magnitude).

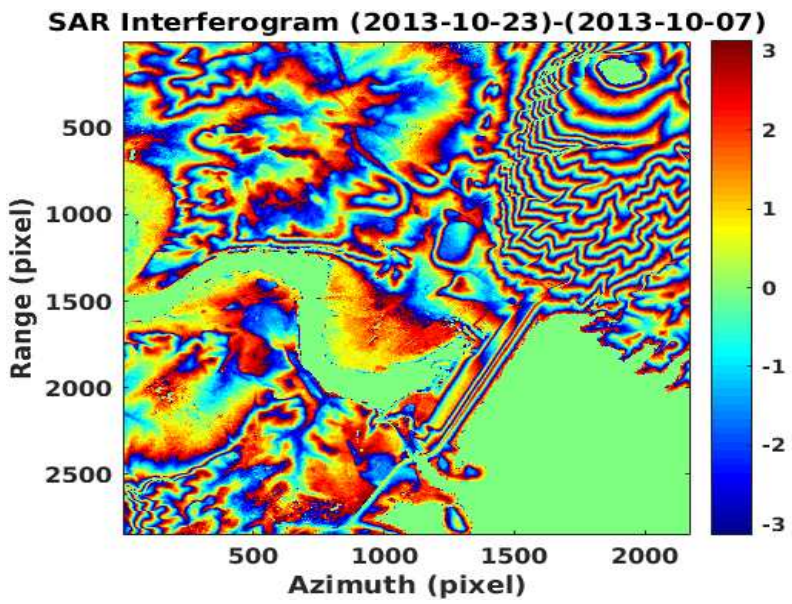

Fig. 6. Wrapped and flattened T1 InSAR fringes. 


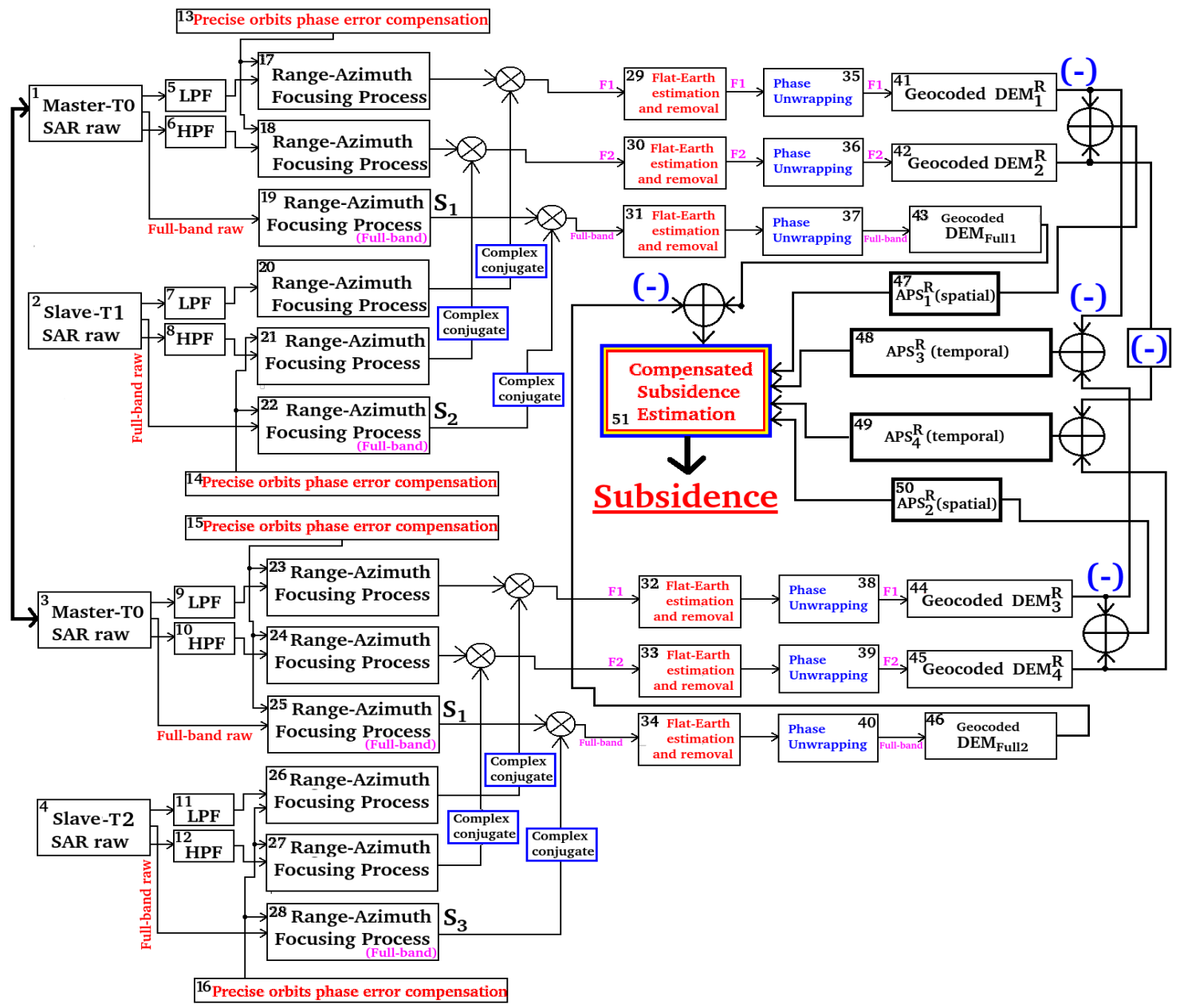

Fig. 3. MCA-APS-DInSAR computational scheme.

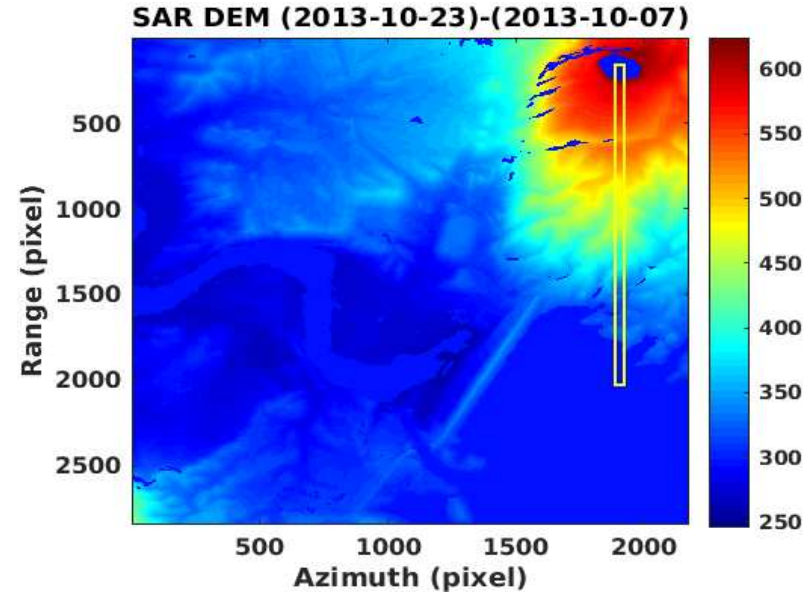

Fig. 7. T1 InSAR estimated DEM.

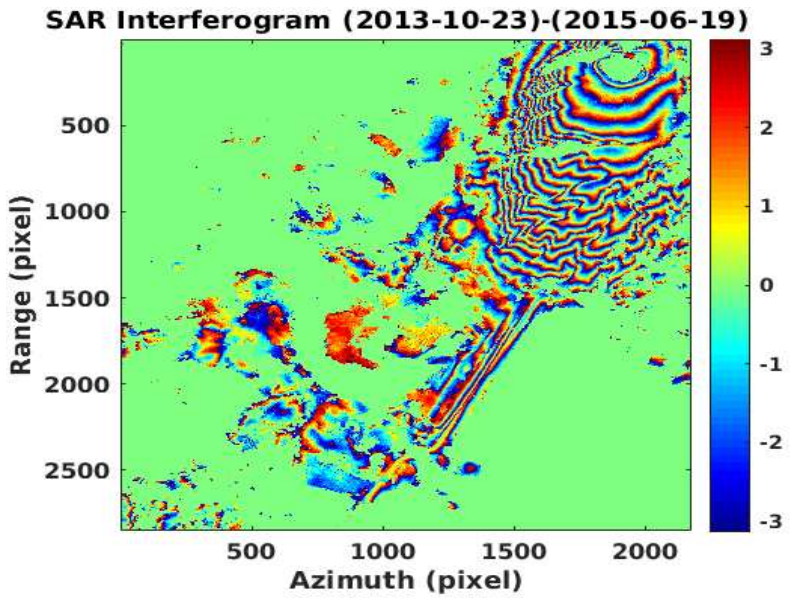

Fig. 8. Wrapped and flattened T1 InSAR fringes. The image is masked at 0.45 of temporal coherence. 


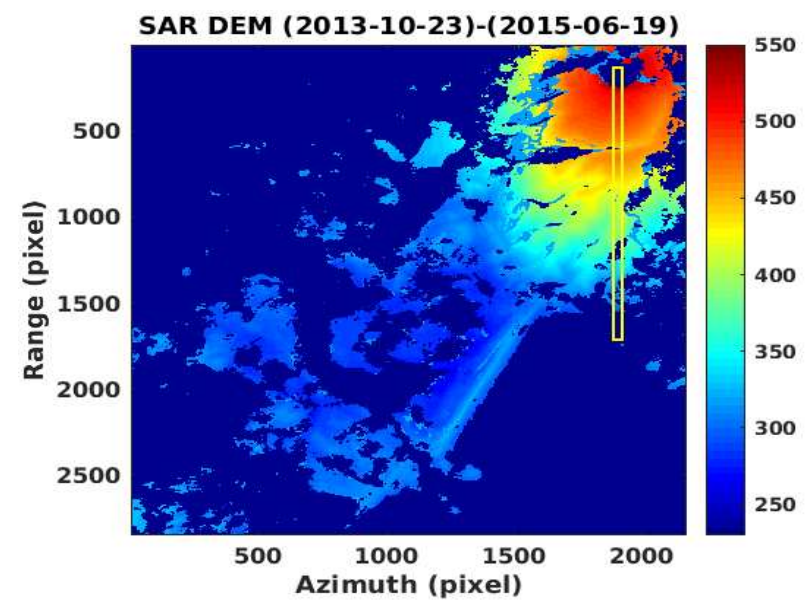

Fig. 9. T2 InSAR estimated DEM.

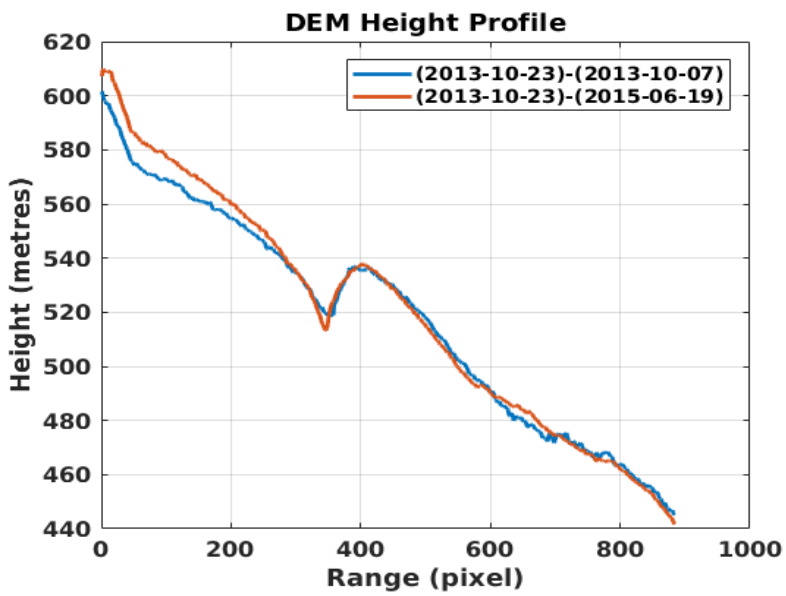

Fig. 10. DEM profiles.

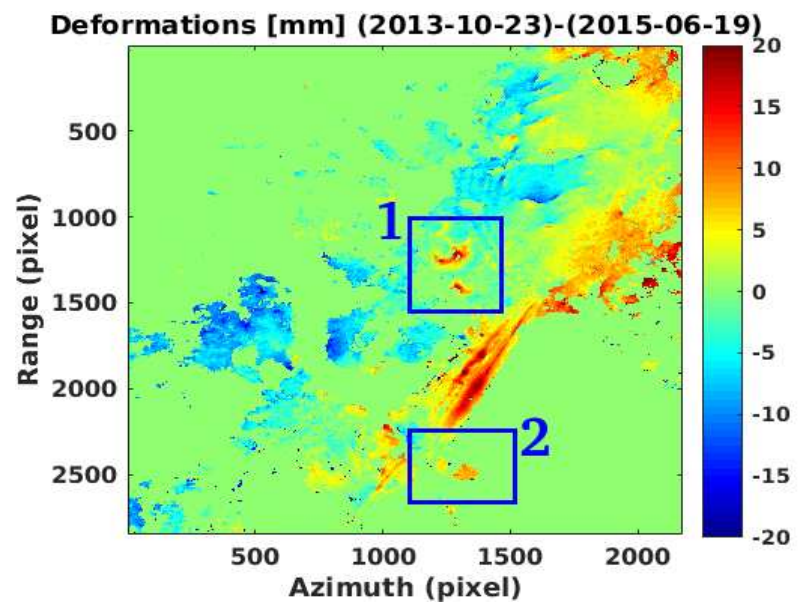

Fig. 11. Uncompensated displacement map.

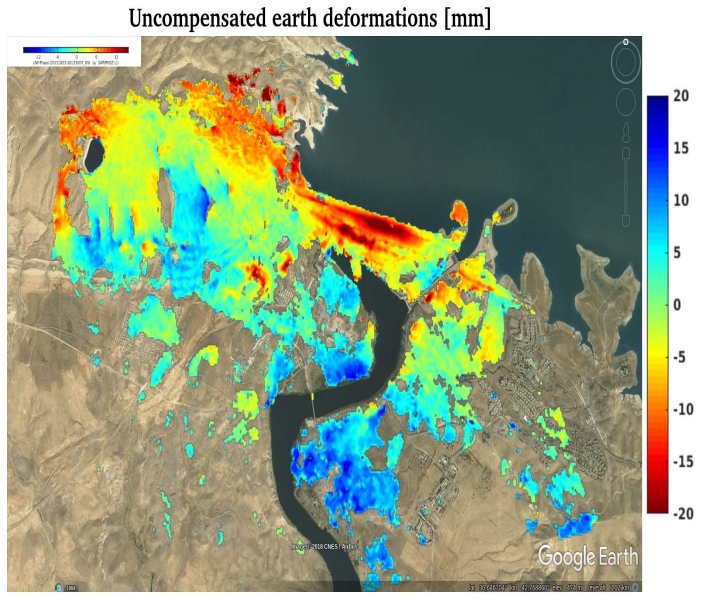

Fig. 12. APS uncompensated orthorectified Earth deformation map.

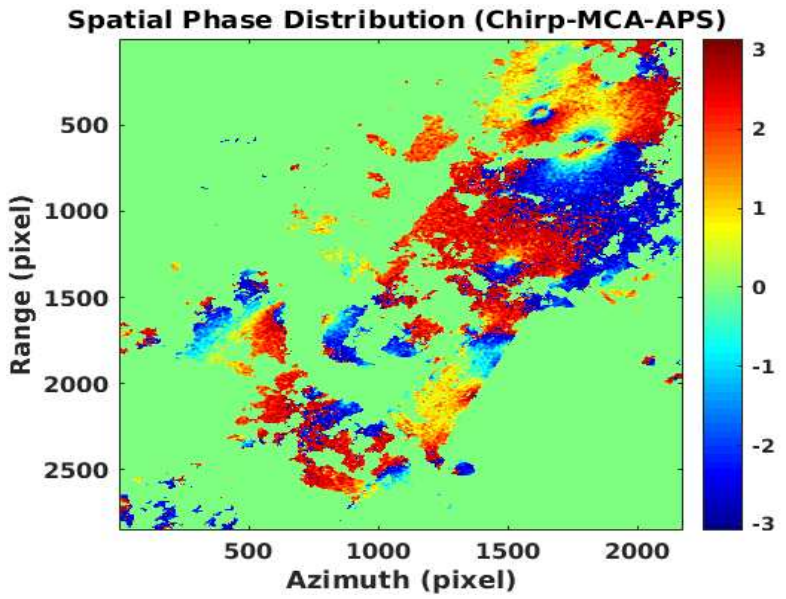

Fig. 13. Atmospheric phase effects for $\operatorname{APS}_{1}^{R}$.

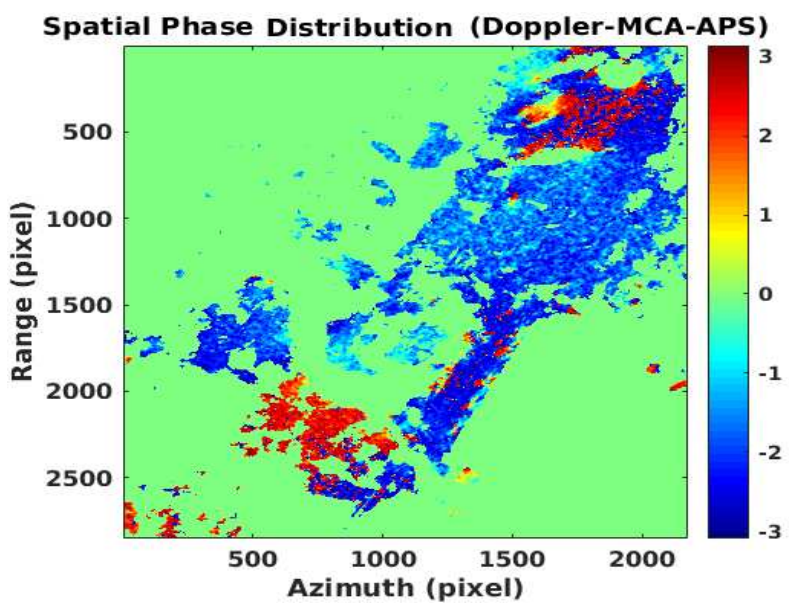

Fig. 14. Atmospheric phase effects for $\operatorname{APS}_{2}^{R}$. 


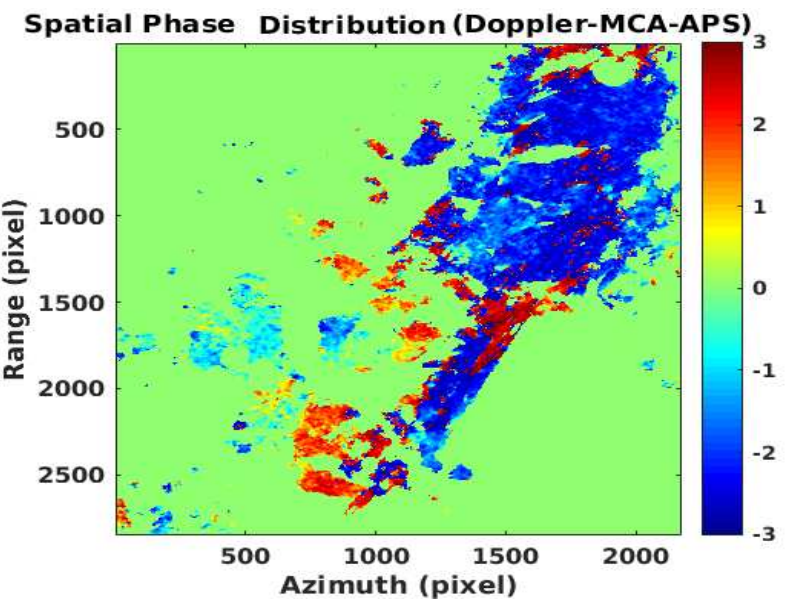

Fig. 15. Atmospheric phase effects for $\operatorname{APS}_{1}^{D}$.

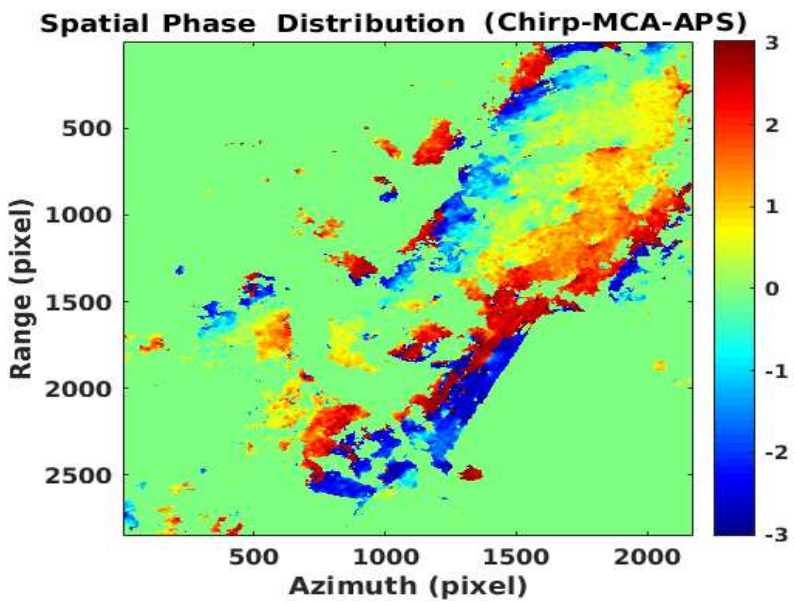

Fig. 16. Atmospheric phase effects for $\operatorname{APS}_{2}^{D}$.

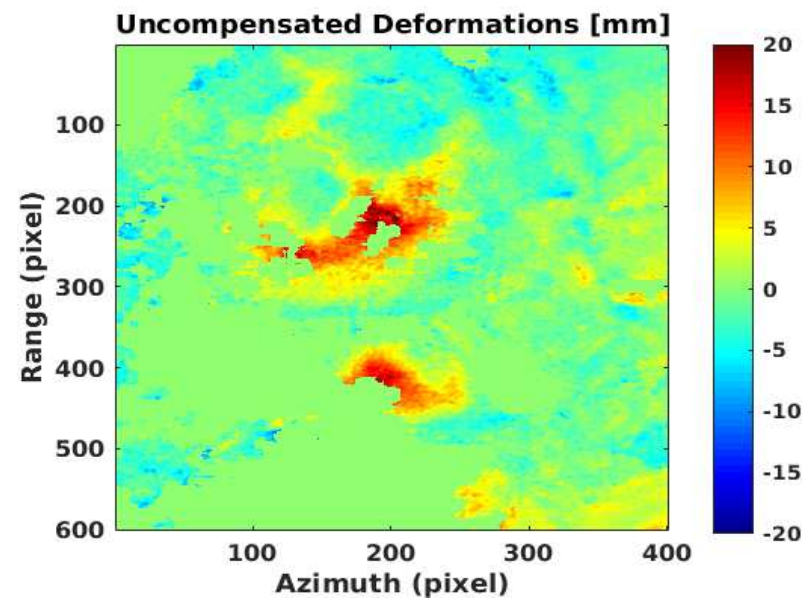

Fig. 17. Uncompensated displacement map.

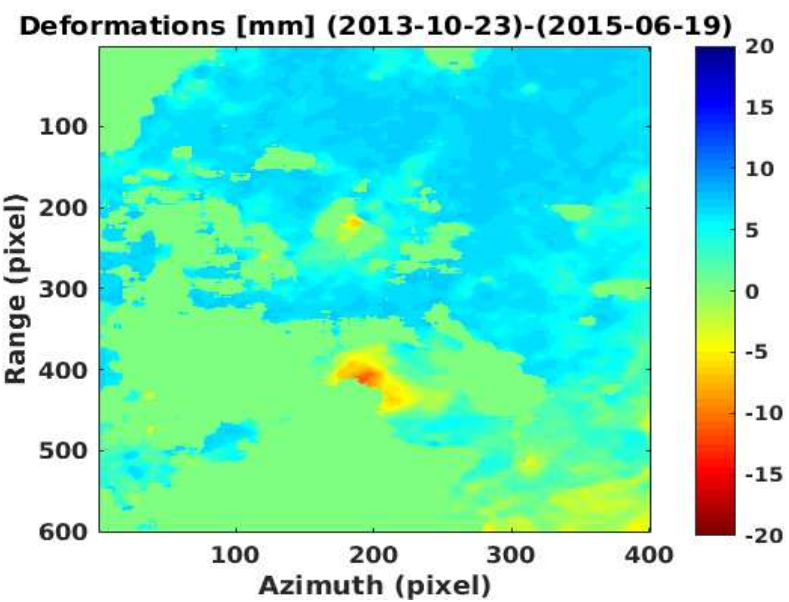

Fig. 18. Compensated displacement map.

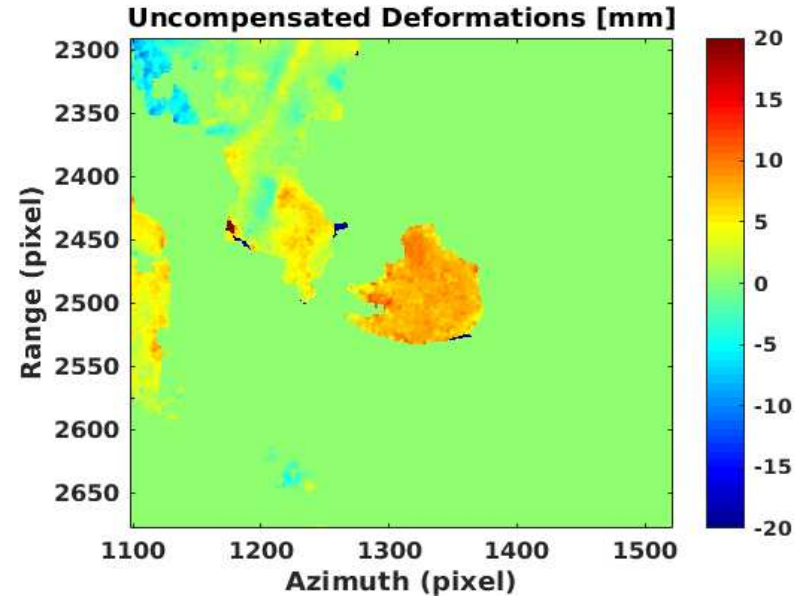

Fig. 19. Uncompensated displacement map.

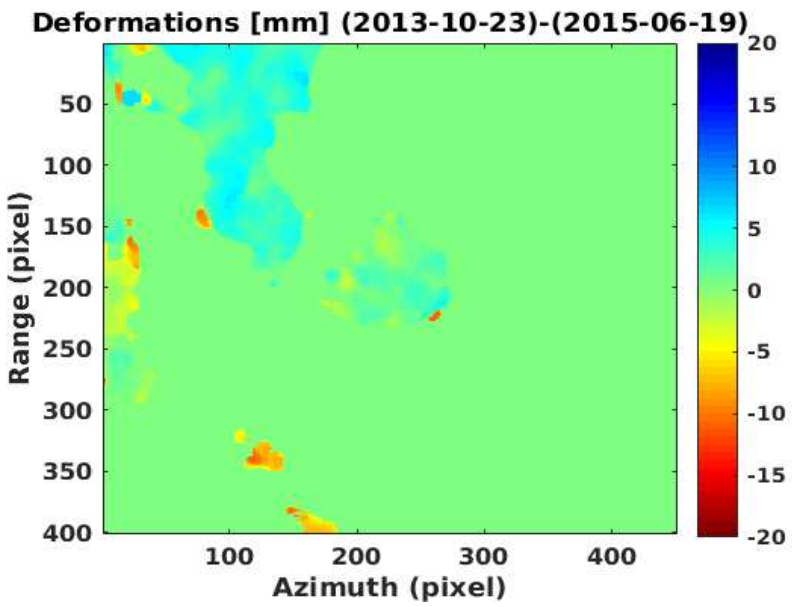

Fig. 20. Compensated displacement map. 


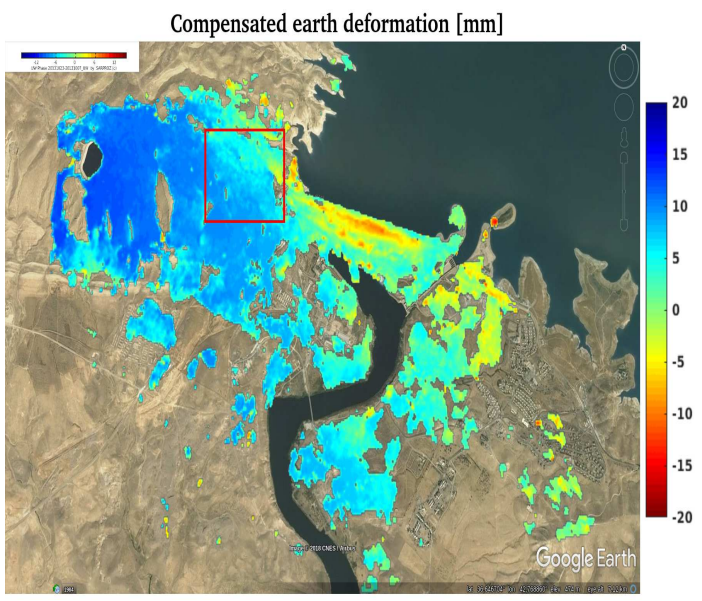

Fig. 21. APS compensated orthorectified Earth deformation map.

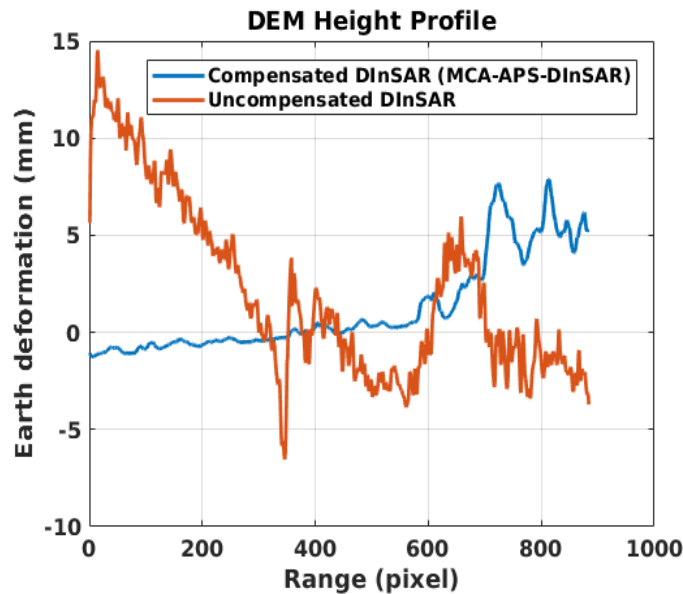

Fig. 22. Uncompensated (orange line) versus compensated (blue line) Earth deformation profiles.

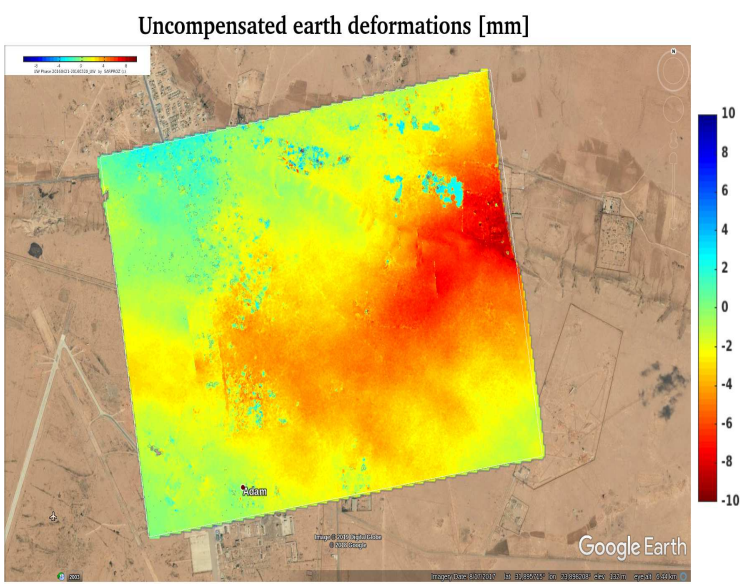

Fig. 23. APS uncompensated orthorectified Earth deformation map.

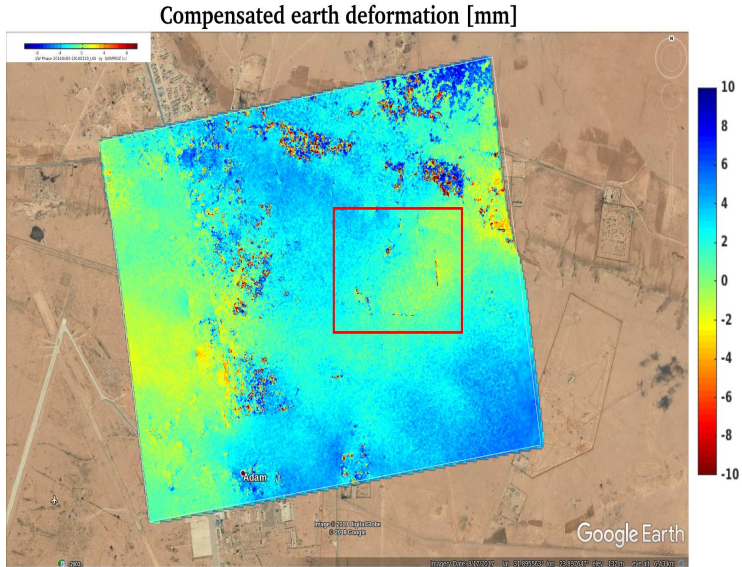

Fig. 24. APS compensated orthorectified Earth deformation map.

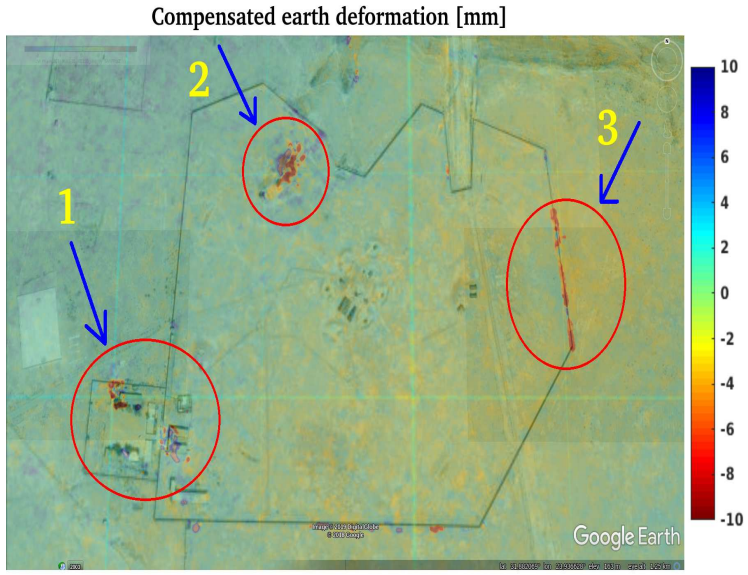

Fig. 25. Red box area of the APS-compensated orthorectified Earth deformation map visible in Fig. 24.

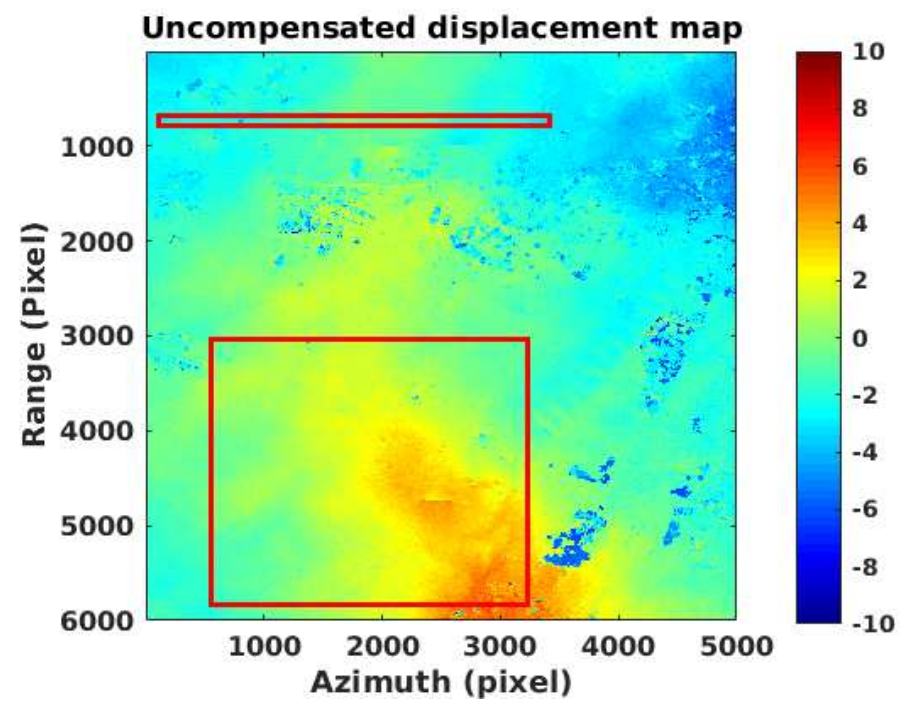

Fig. 26. Effects of band overlap: uncompensated map. 


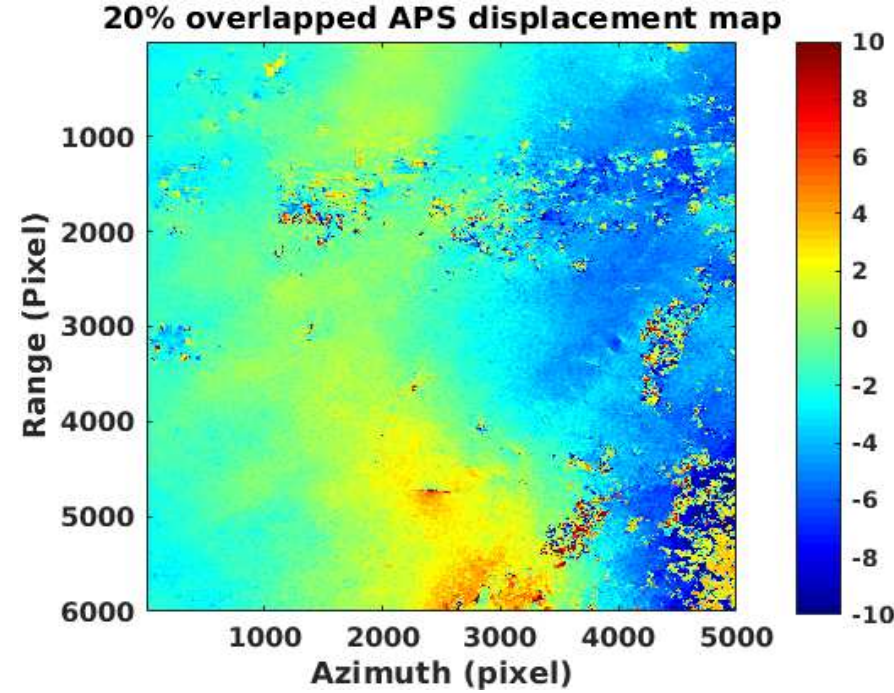

Fig. 27. Effects of band overlap: compensated map with an overlap of about $20 \%$ for the red box region of Fig. 26.

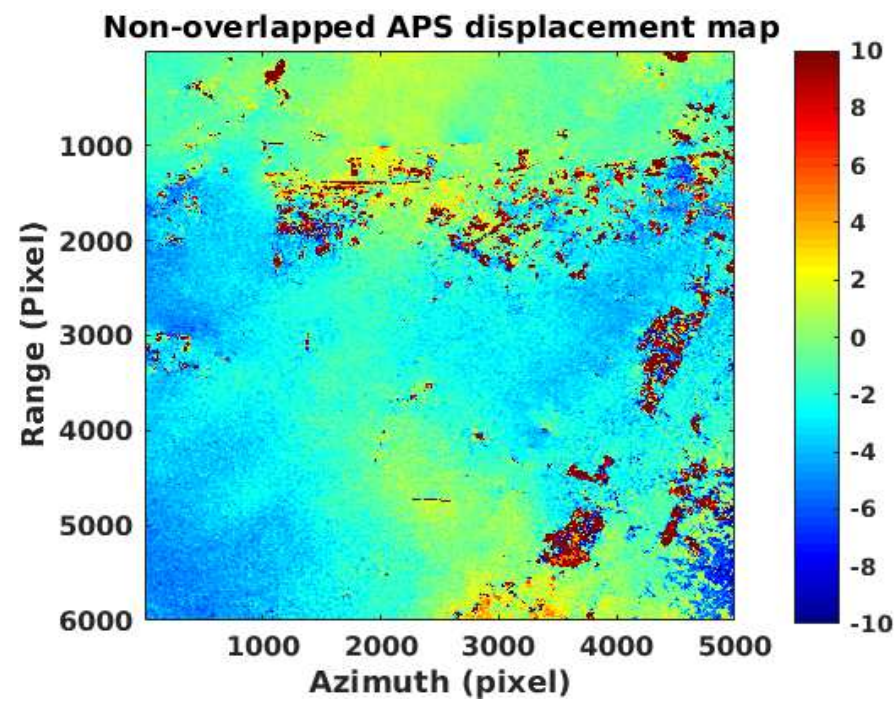

Fig. 28. Effects of band overlap: compensated map without overlap for the red box region of Fig. 26.

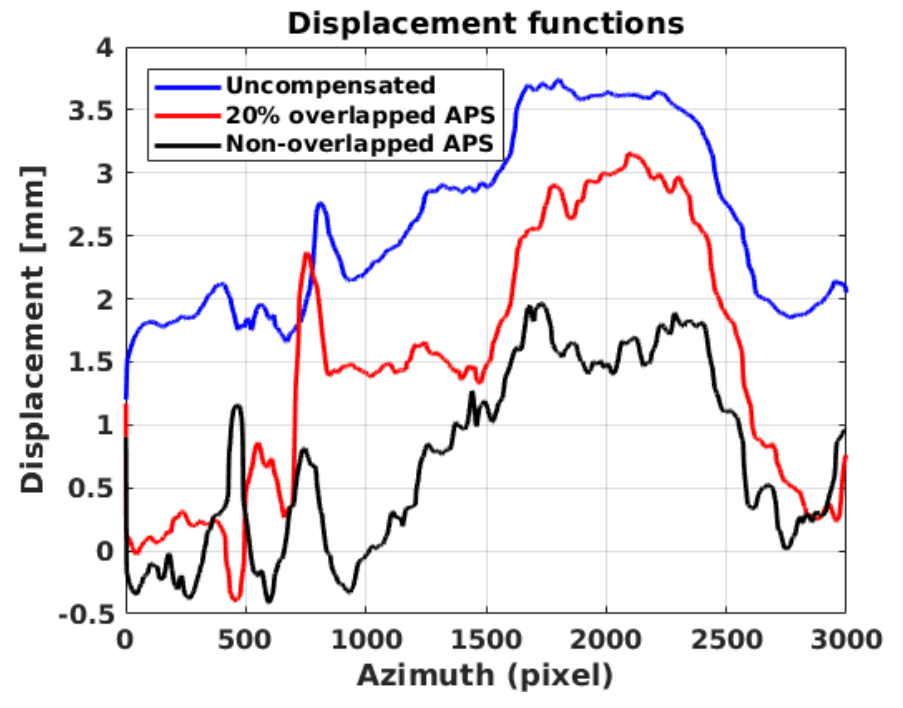

Fig. 29. Effects of band overlap: height profiles for region in the red line of Fig. 26. 